\title{
A locally supersymmetric $\mathrm{SO}(10,2)$ invariant action for $D=12$ supergravity
}

\author{
Leonardo Castellani \\ Dipartimento di Scienze e Innovazione Tecnologica, Università del Piemonte Orientale, \\ viale T. Michel 11, 15121 Alessandria, Italy \\ INFN, Sezione di Torino, \\ via P. Giuria 1, 10125 Torino, Italy \\ Arnold-Regge Center, \\ via P. Giuria 1, 10125 Torino, Italy \\ E-mail: leonardo.castellani@uniupo.it
}

ABSTRACT: We present an action for $N=1$ supergravity in $10+2$ dimensions, containing the gauge fields of the $\operatorname{OSp}(1 \mid 64)$ superalgebra, i.e. one-forms $B^{(n)}$ with $n=1,2,5,6,9,10$ antisymmetric $\mathrm{D}=12$ Lorentz indices and a Majorana gravitino $\psi$. The vielbein and spin connection correspond to $B^{(1)}$ and $B^{(2)}$ respectively. The action is not gauge invariant under the full $\operatorname{OSp}(1 \mid 64)$ superalgebra, but only under a subalgebra $\tilde{F}$ (containing the $F$ algebra $\operatorname{OSp}(1 \mid 32))$, whose gauge fields are $B^{(2)}, B^{(6)}, B^{(10)}$ and the Weyl projected Majorana gravitino $\frac{1}{2}\left(1+\Gamma_{13}\right) \psi$. Supersymmetry transformations are therefore generated by a Majorana-Weyl supercharge and, being part of a gauge superalgebra, close off-shell. The action is simply $\int S \operatorname{Tr}\left(\mathbf{R}^{6} \boldsymbol{\Gamma}\right)$ where $\mathbf{R}$ is the $\operatorname{OSp}(1 \mid 64)$ curvature supermatrix twoform, and $\boldsymbol{\Gamma}$ is a constant supermatrix involving $\Gamma_{13}$ and breaking $\operatorname{OSp}(1 \mid 64)$ to its $\tilde{F}$ subalgebra. The usual Einstein-Hilbert term is included in the action.

KEYwords: Supergravity Models, F-Theory, Field Theories in Higher Dimensions, Supersymmetric Gauge Theory

ArXIV EPRINT: 1705.00638 


\section{Contents}

1 Introduction 1

2 Mac Dowell-Mansouri action revisited 3

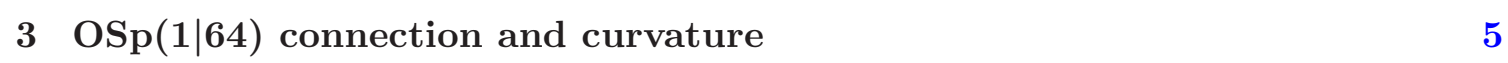

4 The $D=12, N=1$ supergravity action 6

$\begin{array}{llr}5 & \text { Conclusions } & 9\end{array}$

A $\quad \mathrm{D}=12 \Gamma$ matrices, $\mathrm{Sp}(64)$ algebra and a Fierz identity 9

$\begin{array}{ll}\text { B The } \tilde{\boldsymbol{F}} \text { superalgebra } & 11\end{array}$

\section{Introduction}

Supergravity theories in dimensions greater than $D=11$ are believed to be inconsistent, since their reduction to $D=4$ would produce more than $N=8$ supersymmetries, involving multiplets with spin $\geq 2$, and it is known that coupling of gravity with a finite number of higher spins is problematic.

On the other hand a twelve dimensional theory with signature $(10,2)$ avoids this difficulty, since fermions can be both Majorana and Weyl in $D=10+2$, with 32 real components, and therefore giving rise to at most eight supercharges when reduced to $D=4$. This fact has encouraged over the years various attempts and proposals ([1]-[13]) for a twelve-dimensional field theory of supergravity.

A $D=10+2$ structure emerges also from string/brane theory, and has been named $F$-theory [14]. The $\operatorname{OSp}(1 \mid 32)$ superalgebra, a natural choice for the gauge algebra of a $D=10+2$ supergravity, is also called $F$ algebra [13].

Supergravity theories in various dimensions have been constructed using different approaches, such as superspace (see for ex. [15-17]), group geometric [18-22], ChernSimons [23], component (or "Noether") techniques (for ex. [17, 24]), or combinations thereof (for ex. [25]).

Here we follow the "gauge supergravity" road, inspired by Chern-Simons supergravities [23], and adapted to even dimensions via a higher curvature generalization of the Mac Dowell-Mansouri (MDM) action [26]. In the MDM framework (see also [28]) the fields of $N=1, D=4$ supergravity are seen as parts of the $\operatorname{OSp}(1 \mid 4)$ superconnection, as originally proposed in [27], and enter the MDM action only via the $\operatorname{OSp}(1 \mid 4)$ curvature. The MDM action however is not invariant under $\mathrm{OSp}(1 \mid 4)$ gauge transformations: indeed, besides the $\operatorname{OSp}(1 \mid 4)$ curvature, it also contains a constant matrix involving $\gamma_{5}$, that breaks $\operatorname{OSp}(1 \mid 4)$ 
to its Lorentz subalgebra. Since all supertranslations are broken, one may wonder how could this action be locally supersymmetric. In fact one finds that it is supersymmetric in second order formalism (i.e. expressing the spin connection in terms of the vielbein and the gravitino via the supertorsion constraint). But also remaining in first order formalism supersymmetry can be restored by modifying the supersymmetry transformation law of the spin connection. The MDM action, after dispensing with topological terms, becomes the action of (anti)de Sitter $N=1$ supergravity in $D=4$. Note that supersymmetry here is not gauge supersymmetry, since it is not part of a superalgebra of transformations. There is no guarantee that the algebra of the "restored supersymmetry" closes off-shell, and indeed for this one needs auxiliary fields.

This being the state of affairs in $D=4$, can we do something similar in $D=12$ dimensions? The present paper provides an affirmative answer. The superalgebra $\operatorname{OSp}(1 \mid 4)$ is replaced by $\operatorname{OSp}(1 \mid 64)$, the corresponding "would be" gauge fields being one-forms $B^{(n)}$ with $n=1,2,5,6,9,10$ antisymmetric Lorentz indices and a Majorana gravitino $\psi$. The vielbein and the spin connection are identified with $B^{(1)}$ and $B^{(2)}$ respectively. These one-forms are organized into an $\operatorname{OSp}(1 \mid 64)$ connection, in an explicit $65 \times 65$ dimensional supermatrix representation. The $D=12$ action is constructed using exclusively the $\operatorname{OSp}(1 \mid 64)$ supermatrix curvature, and a constant matrix involving $\Gamma_{13}$. This constant matrix ensures that the action is not topological (similarly to the MDM action) and breaks OSp(1|64) to a subalgebra $\tilde{F}=\operatorname{OSp}(1 \mid 32) \oplus \mathrm{Sp}(32)$, under which the action is invariant. ${ }^{1}$ Here part of the supersymmetry of $\operatorname{OSp}(1 \mid 64)$ survives, in contrast to the $D=4$ case. Supersymmetry is then a gauge symmetry, and closes off-shell. Twelve dimensional Lorentz symmetry $\mathrm{SO}(10,2)$ is also part of the $\tilde{F}$ gauge symmetry, so that the action is $\mathrm{SO}(10,2)$ invariant.

Under the action of $\tilde{F}$, the $\operatorname{OSp}(1 \mid 64)$ fields split into a gauge multiplet and a matter multiplet. The gauge multiplet contains the $\tilde{F}$ gauge fields: the spin connection $B^{(2)}$, a Majorana-Weyl gravitino $\psi_{+}$, and the other "even" one-form fields $B^{(6)}$ and $B^{(10)}$. The matter multiplet contains the remaining $\operatorname{OSp}(1 \mid 64)$ fields: the "odd" one-form fields $B^{(1)}$ (the vielbein), $B^{(5)}$ and $B^{(9)}$, and a Majorana anti-Weyl gravitino $\psi_{-}$.

Finally, let us emphasize that by " $D=12$ supergravity" we really mean a field theory in $10+2$ dimensions, described by a geometrical action (the integral of a 12-form Lagrangian), containing the usual Einstein-Hilbert term and invariant under a gauge algebra that includes supersymmetry. This is much in the spirit of Chern-Simons supergravities in odd dimensions [29]. The implications of the field equations for the correct counting of degrees of freedom are still to be investigated.

The plan of the paper is as follows. Section 2 is a revisitation of the Mac DowellMansouri action and its symmetries. Section 3 establishes notations for the $\operatorname{OSp}(1 \mid 64)$ superalgebra, its connection and curvature components. In section 4 the $D=12, N=1$ supergravity action is presented, and the proof of its invariance under $\tilde{F}$ gauge transformations is provided. Field transformation rules are given, and field equations are briefly discussed. Section 5 contains some conclusions and outlook.

\footnotetext{
${ }^{1}$ The $\tilde{F}$ algebra contains the $F$ algebra: in fact the $F$ algebra is the $\operatorname{OSp}(1 \mid 32)$ part of $\tilde{F}$.
} 


\section{Mac Dowell-Mansouri action revisited}

This section is just a review, and is included here because of its similarity to the $D=12$ construction. Most of it is taken from ref. [30].

The Mac Dowell-Mansouri action [26] is a $R^{2}$-type reformulation of (anti)de Sitter supergravity in $D=4$. It is based on the supergroup $\operatorname{OSp}(1 \mid 4)$, and the fields $V^{a}$ (vierbein), $\omega^{a b}$ (spin connection) and $\psi$ (gravitino $^{2}$ ) are 1-forms contained in the $\operatorname{OSp}(1 \mid 4)$ connection $\boldsymbol{\Omega}$, in a $5 \times 5$ supermatrix representation:

$$
\boldsymbol{\Omega} \equiv\left(\begin{array}{cc}
\Omega & \psi \\
\bar{\psi} & 0
\end{array}\right), \quad \Omega \equiv \frac{1}{4} \omega^{a b} \gamma_{a b}-\frac{i}{2} V^{a} \gamma_{a}
$$

The corresponding $\operatorname{OSp}(1 \mid 4)$ curvature supermatrix is

$$
\mathbf{R}=d \boldsymbol{\Omega}-\boldsymbol{\Omega} \wedge \boldsymbol{\Omega} \equiv\left(\begin{array}{cc}
R & \Sigma \\
\bar{\Sigma} & 0
\end{array}\right)
$$

and straightforward matrix algebra yields: ${ }^{3}$

$$
\begin{aligned}
R & =\frac{1}{4} R^{a b} \gamma_{a b}-\frac{i}{2} R^{a} \gamma_{a} \\
\Sigma & =d \psi-\frac{1}{4} \omega^{a b} \gamma_{a b} \psi+\frac{i}{2} V^{a} \gamma_{a} \psi \\
\bar{\Sigma} & =d \bar{\psi}-\frac{1}{4} \bar{\psi} \omega^{a b} \gamma_{a b}+\frac{i}{2} \bar{\psi} V^{a} \gamma_{a} \\
R^{a b} & \equiv d \omega^{a b}-\omega^{a}{ }_{c} \omega^{c b}+V^{a} V^{b}+\frac{1}{2} \bar{\psi} \gamma^{a b} \psi \\
R^{a} & \equiv d V^{a}-\omega^{a}{ }_{b} V^{b}-\frac{i}{2} \bar{\psi} \gamma^{a} \psi
\end{aligned}
$$

We have also used the Fierz identity for 1-form Majorana spinors:

$$
\psi \bar{\psi}=\frac{1}{4}\left(\bar{\psi} \gamma^{a} \psi \gamma_{a}-\frac{1}{2} \bar{\psi} \gamma^{a b} \psi \gamma_{a b}\right)
$$

(to prove it, just multiply both sides by $\gamma_{c}$ or $\gamma_{c d}$ and take the trace on spinor indices).

The Mac Dowell-Mansouri action can be written in terms of the $\operatorname{OSp}(1 \mid 4)$ curvature $\mathbf{R}$ as:

$$
S=4 \int S T r(\mathbf{R G R \Gamma})
$$

where $S \operatorname{Tr}$ is the supertrace and $\mathbf{G}, \boldsymbol{\Gamma}$ are the following constant matrices:

$$
\boldsymbol{\Gamma} \equiv\left(\begin{array}{cc}
i \gamma_{5} & 0 \\
0 & 0
\end{array}\right), \quad \mathbf{G}=\mathbf{1}+\frac{\boldsymbol{\Gamma}^{2}}{2}=\frac{1}{2}\left(\begin{array}{ll}
1 & 0 \\
0 & 2
\end{array}\right)
$$

\footnotetext{
${ }^{2}$ The gravitino $\psi$ is a Majorana spinor, i.e. $\bar{\psi}=\psi^{T} C$, where $C$ is the charge conjugation matrix.

${ }^{3} \mathrm{We}$ omit wedge products between forms.
} 
All boldface quantities are $5 \times 5$ supermatrices. Carrying out the supertrace, and then the spinor trace, leads to the familiar expression of the MacDowell-Mansouri action:

$$
S=2 i \int \operatorname{Tr}\left(R \wedge R \gamma_{5}+2 \Sigma \wedge \bar{\Sigma} \gamma_{5}\right)=2 \int \frac{1}{4} R^{a b} \wedge R^{c d} \varepsilon_{a b c d}-2 i \bar{\Sigma} \wedge \gamma_{5} \Sigma
$$

After inserting the curvature definitions the action takes the form

$$
S=\int \mathcal{R}^{a b} V^{c} V^{d} \varepsilon_{a b c d}+4 \bar{\rho} \gamma_{a} \gamma_{5} \psi V^{a}+\frac{1}{2}\left(V^{a} V^{b} V^{c} V^{d}+2 \bar{\psi} \gamma^{a b} \psi V^{c} V^{d}\right) \epsilon_{a b c d}
$$

with

$$
\mathcal{R}^{a b} \equiv d \omega^{a b}-\omega^{a}{ }_{c} \omega^{c b}, \quad \rho \equiv d \psi-\frac{1}{4} \omega^{a b} \gamma_{a b} \psi \equiv \mathcal{D} \psi
$$

We have dropped the topological term $\mathcal{R}^{a b} \mathcal{R}^{c d} \epsilon_{a b c d}$ (Euler form), and used the gravitino Bianchi identity

$$
\mathcal{D} \rho=-\frac{1}{4} \mathcal{R}^{a b} \gamma_{a b} \psi
$$

and the gamma matrix identity $2 \gamma_{a b} \gamma_{5}=i \epsilon_{a b c d} \gamma^{c d}$ to recognize that $\frac{1}{2} \mathcal{R}^{a b} \bar{\psi} \gamma^{c d} \psi \epsilon_{a b c d}-$ $4 i \bar{\rho} \gamma_{5} \rho$ is a total derivative. The action (2.12) describes $N=1, D=4$ anti-De Sitter supergravity, the last term being the supersymmetric cosmological term. After rescaling the vielbein and the gravitino as $V^{a} \rightarrow \lambda V^{a}, \psi \rightarrow \sqrt{\lambda} \psi$ and dividing the action by $\lambda^{2}$, the usual (Minkowski) $N=1, D=4$ supergravity is retrieved by taking the limit $\lambda \rightarrow 0$. This corresponds to the Inonü-Wigner contraction of $\operatorname{OSp}(1 \mid 4)$ to the superPoincaré group.

Invariances. As is well known, the action (2.9), although a bilinear in the $\operatorname{OSp}(1 \mid 4)$ curvature, is not invariant under the $\operatorname{OSp}(1 \mid 4)$ gauge transformations:

$$
\delta_{\epsilon} \boldsymbol{\Omega}=d \boldsymbol{\epsilon}-\boldsymbol{\Omega} \boldsymbol{\epsilon}+\boldsymbol{\epsilon} \Longrightarrow \delta_{\boldsymbol{\epsilon}} \mathbf{R}=-\mathbf{R} \boldsymbol{\epsilon}+\boldsymbol{\epsilon} \mathbf{R}
$$

where $\boldsymbol{\epsilon}$ is the $\operatorname{OSp}(1 \mid 4)$ gauge parameter:

$$
\boldsymbol{\epsilon} \equiv\left(\begin{array}{cr}
\frac{1}{4} \varepsilon^{a b} \gamma_{a b}-\frac{i}{2} \varepsilon^{a} \gamma_{a} \epsilon \\
\bar{\epsilon} & 0
\end{array}\right)
$$

In fact it is not a Yang-Mills action (involving the exterior product of $\mathbf{R}$ with its Hodge dual), nor a topological action $\int S \operatorname{Tr}(\mathbf{R R})$ : the constant supermatrices $\mathbf{G}$ and $\boldsymbol{\Gamma}$ ruin the $\operatorname{OSp}(1 \mid 4)$ gauge invariance, and break it to its Lorentz subgroup. Indeed the gauge variation of the action $(2.9)$

$$
\delta S=4 \int S \operatorname{Tr}(\mathbf{R}[\mathbf{G}, \boldsymbol{\epsilon}] \mathbf{R} \boldsymbol{\Gamma}+\mathbf{R G R}[\boldsymbol{\Gamma}, \boldsymbol{\epsilon}])
$$

vanishes when $\boldsymbol{\epsilon}$ commutes with $\boldsymbol{\Gamma}$ (and therefore with $\mathbf{G}$ ), and this happens only when $\boldsymbol{\epsilon}$ in (2.16) has $\varepsilon^{a}=\epsilon=0$, so that only Lorentz rotations leave the action invariant.

Specializing the gauge parameter $\epsilon$ to describe supersymmetry variations (i.e. only $\epsilon \neq 0$ in (2.16)), eq. (2.17) yields the supersymmetry variation of the Mac Dowell-Mansouri action:

$$
\begin{aligned}
\delta_{\text {susy }} S & =2 i \int\left(\bar{\epsilon}\left[\gamma_{5}, R\right] \Sigma+\bar{\Sigma}\left[\gamma_{5}, R\right] \epsilon\right) \\
& =-4 \int R^{a} \bar{\Sigma} \gamma_{a} \gamma_{5} \epsilon
\end{aligned}
$$


with $R$ defined in (2.3). This variation is proportional to the torsion $R^{a}$, since only $R^{a} \gamma_{a}$ in $R$ has a nonzero commutator with $\gamma_{5}$. Therefore in second-order formalism, i.e. using the torsion constraint $R^{a}=0$ to express $\omega^{a b}$ in terms of $V^{a}$ and $\psi$, the action is indeed supersymmetric. Another way to recover supersymmetry is by modifying the supersymmetry variation of the spin connection, see for ex. [28]. In both cases supersymmetry is not part of a gauge superalgebra: off-shell closure of the supersymmetry transformations is not automatic, and indeed necessitates the introduction of auxiliary fields.

We shall now jump to 12 dimensions, and write a geometrical $\mathbf{R}^{6}$-type action that resembles the $\mathbf{R}^{2}$-type Mac Dowell-Mansouri action of $D=4$. The action will be invariant under the $\tilde{F}$ subalgebra of $\operatorname{OSp}(1 \mid 64)$. Contrary to the $D=4$ case, $N=1$ supersymmetry (with a Majorana-Weyl supercharge) survives as part of the $\tilde{F}$ algebra, and closes off-shell.

\section{$3 \quad \operatorname{OSp}(1 \mid 64)$ connection and curvature}

The algebra. The $\operatorname{OSp}(1 \mid 64)$ superalgebra has the following structure:

$$
\begin{aligned}
{\left[Z_{(n)}, Z_{(m)}\right] } & =c_{(n)(m)}^{(r)} Z_{(r)} \\
\left\{\bar{Q}_{\alpha}, \bar{Q}_{\beta}\right\} & =\frac{1}{16} \frac{(-1)^{n}}{n !}\left(C \Gamma^{(n)}\right)_{\alpha \beta} Z_{(n)} \\
{\left[Z_{(n)}, \bar{Q}_{\alpha}\right] } & =\frac{1}{2}\left(\Gamma_{(n)}\right)_{\alpha}^{\beta} \bar{Q}_{\beta}
\end{aligned}
$$

where the bosonic generators $Z_{(n)}$ with $n=1,2,5,6,9,10$ have $n$ antisymmetrized Lorentz indices and are dual to the one-forms $B^{(n)}$, and the Majorana supersymmetry charge $\bar{Q}$ is dual to the Majorana gravitino one-form $\psi$. In (3.2) and (3.3) the $\mathrm{D}=12$ gamma matrices $\Gamma^{(n)}$ with $n$ antisymmetrized indices appear as structure constants, and $C$ is the charge conjugation matrix. The structure constants $c_{(n)(m)}^{(r)}$ in (3.1) are obtained from the commutation relations of the $\Gamma^{(n)}$, and are given in appendix A. Repeated $(n)$ indices are summed on $n=1,2,5,6,9,10$.

The $65 \times 65$ supermatrix representation. Gamma matrices in $D=12$ have a $64 \times 64$ matrix representation and the superalgebra above can be realized by a $65 \times 65$ supermatrix representation:

$$
Z_{(n)}=\left(\begin{array}{cc}
\frac{1}{2} \Gamma_{(n)} & 0 \\
0 & 0
\end{array}\right), \quad \bar{Q}_{\alpha}=Q^{\beta} C_{\beta \alpha}=\left(\begin{array}{cc}
0 & \delta_{\alpha}^{\rho} \\
C_{\sigma \alpha} & 0
\end{array}\right)
$$

To verify the anticommutations (3.2), one needs the identity

$$
\delta_{\alpha}^{\rho} C_{\sigma \beta}+\delta_{\beta}^{\rho} C_{\sigma \alpha}=\frac{1}{32} \frac{(-1)^{n}}{n !}\left(C \Gamma_{(n)}\right)_{\alpha \beta}\left(\Gamma^{(n)}\right)_{\sigma}^{\rho}
$$

deducible from the Fierz identity (A.10) by factoring out the two spinor Majorana oneforms. 
Connection and curvature. The 1-form $\operatorname{OSp}(1 \mid 64)$-connection is given by

$$
\boldsymbol{\Omega}=\frac{1}{n !} B^{(n)} Z_{(n)}+\bar{Q}_{\alpha} \psi^{\alpha}
$$

In the $65 \times 65$ supermatrix representation:

$$
\boldsymbol{\Omega} \equiv\left(\begin{array}{cc}
\Omega & \psi \\
-\bar{\psi} & 0
\end{array}\right), \quad \Omega=\frac{1}{2 \cdot n !} B^{(n)} \Gamma_{(n)}
$$

The corresponding curvature supermatrix 2 -form is

$$
\mathbf{R}=d \boldsymbol{\Omega}-\boldsymbol{\Omega} \wedge \boldsymbol{\Omega} \equiv\left(\begin{array}{cc}
R & \Sigma \\
-\bar{\Sigma} & 0
\end{array}\right)
$$

with $^{4}$

$$
\begin{aligned}
& R=d \Omega-\Omega \Omega+\psi \bar{\psi} \equiv \frac{1}{2 \cdot n !} R^{(n)} \Gamma_{(n)} \\
& \Sigma=d \psi-\Omega \psi=d \psi-\frac{1}{2 \cdot n !} B^{(n)} \Gamma_{(n)} \psi \\
& \bar{\Sigma}=d \bar{\psi}-\bar{\psi} \Omega=d \bar{\psi}-\frac{1}{2 \cdot n !} \bar{\psi} B^{(n)} \Gamma_{(n)}
\end{aligned}
$$

The bosonic curvature components $R^{(n)}$ are easily determined:

$$
R^{(n)}=d B^{(n)}+\frac{1}{2} c_{(m)(r)}^{(n)} B^{(m)} B^{(r)}-\frac{(-1)^{n}}{32} \bar{\psi} \Gamma^{(n)} \psi
$$

where we used the Fierz identity for 1-form Majorana spinors in (A.10). For example for $R^{(2)}$ we find:

$$
\begin{aligned}
R^{a b}= & d B^{a b}-B^{a c} B_{c}{ }^{b}-V^{a} V^{b}-\frac{1}{32} \bar{\psi} \Gamma^{a b} \psi \\
& -\frac{1}{4 !} B^{c_{1}-c_{4} a} B_{c_{1}-c_{4}}{ }^{b}+\frac{1}{5 !} B^{c_{1}-c_{5} a} B_{c_{1}-c_{5}}{ }^{b}-\frac{1}{8 !} B^{c_{1}-c_{8} a} B_{c_{1}-c_{8}} b+\frac{1}{9 !} B^{c_{1}-c_{9} a} B_{c_{1}-c_{9}} b
\end{aligned}
$$

\section{The $D=12, N=1$ supergravity action}

An $\tilde{F}$-invariant action for the fields contained in the $\operatorname{OSp}(1 \mid 64)$ connection can be written as the integral of a Lagrangian 12-form, using the $\operatorname{OSp}(1 \mid 64)$ curvature supermatrix $\mathbf{R}$ :

$$
S=\int S T r\left(\mathbf{R}^{6} \boldsymbol{\Gamma}\right)
$$

where $S \operatorname{Tr}$ is the supertrace and $\boldsymbol{\Gamma}$ is the constant matrix

$$
\boldsymbol{\Gamma} \equiv\left(\begin{array}{cc}
\Gamma_{13} & 0 \\
0 & 1
\end{array}\right)
$$

\footnotetext{
${ }^{4}$ We omit wedge products between forms.
} 
Invariances. Under the $\operatorname{OSp}(1 \mid 64)$ gauge transformations:

$$
\delta_{\boldsymbol{\epsilon}} \boldsymbol{\Omega}=d \boldsymbol{\epsilon}-\boldsymbol{\Omega} \boldsymbol{\epsilon}+\boldsymbol{\epsilon} \boldsymbol{\Omega} \Longrightarrow \delta_{\boldsymbol{\epsilon}} \mathbf{R}=-\mathbf{R} \boldsymbol{\epsilon}+\boldsymbol{\epsilon} \mathbf{R}
$$

where $\boldsymbol{\epsilon}$ is the $\operatorname{OSp}(1 \mid 64)$ gauge parameter:

$$
\boldsymbol{\epsilon} \equiv\left(\begin{array}{ll}
\frac{1}{2 \cdot n !} \varepsilon^{(n)} \Gamma_{(n)} & \epsilon \\
-\bar{\epsilon} & 0
\end{array}\right)
$$

the action (4.1) varies as

$$
\delta S=\int S T r\left(\mathbf{R}^{6}[\boldsymbol{\Gamma}, \boldsymbol{\epsilon}]\right)
$$

Now

$$
[\boldsymbol{\Gamma}, \boldsymbol{\epsilon}]=\left(\begin{array}{cc}
\frac{1}{2 \cdot n !} \varepsilon^{(n)}\left[\Gamma_{13}, \Gamma_{(n)}\right] & \left(\Gamma_{13}-1\right) \epsilon \\
\bar{\epsilon}\left(\Gamma_{13}-1\right) & 0
\end{array}\right)
$$

vanishes when the gauge parameters $\varepsilon^{(n)}$ have even $n$, so that $\Gamma_{(n)}$ commutes with $\Gamma_{13}$, and $\epsilon$ is a Weyl spinor (besides being Majorana), i.e. $\Gamma_{13} \epsilon=\epsilon$. These restrictions on the gauge parameters determine a subalgebra of $\operatorname{OSp}(1 \mid 64)$, generated by $Z_{(2)}, Z_{(6)}, Z_{(10)}$, and $\bar{Q}_{\alpha} P_{+}$, where $P_{+}=\frac{1}{2}\left(1+\Gamma_{13}\right)$. These generators close on the $\tilde{F}=\operatorname{OSp}(1 \mid 32) \oplus \operatorname{Sp}(32)$ subalgebra of $\operatorname{OSp}(1 \mid 64)$.

Note: the analogous action in four dimensions $\int \operatorname{STr}\left(\mathbf{R}^{2} \boldsymbol{\Gamma}\right)$, where $\boldsymbol{\Gamma}$ is as in (4.2) with $\Gamma_{13}$ substituted by $\gamma_{5}$, is not invariant under (chiral) gauge supersymmetry since the supersymmetry parameter cannot be Majorana-Weyl in $D=3+1$.

The $F$ algebra is contained in $\tilde{F}=\operatorname{OSp}(1 \mid 32) \oplus \mathrm{Sp}(32)$, and corresponds to the $\operatorname{OSp}(1 \mid 32)$ term in the direct sum. Its generators are given by $Z_{(2)}^{+} \equiv Z_{(2)} \mathbf{P}_{+}$(66), $Z_{(6)}^{+} \equiv Z_{(6)} \mathbf{P}_{+}\left(462\right.$, selfdual) and $\bar{Q}_{\alpha}^{+} \equiv \bar{Q}_{\alpha} \mathbf{P}_{+}(32)$, where the number of the generators is given between parentheses, and

$$
\mathbf{P}_{ \pm} \equiv\left(\begin{array}{cc}
\frac{1}{2}\left(1 \pm \Gamma_{13}\right) & 0 \\
0 & 0
\end{array}\right)
$$

The $\mathrm{Sp}(32)$ part of $\tilde{F}$ is generated by $Z_{(2)}^{-} \equiv Z_{(2)} \mathbf{P}_{-}(66)$ and $Z_{(6)}^{-} \equiv Z_{(6)} \mathbf{P}_{-}$(462, antiselfdual). The $Z_{(10)}$ generators of $\tilde{F}$ are absorbed into $Z_{(2)} \mathbf{P}_{ \pm}$since $\Gamma_{(2)} \Gamma_{13}$ is proportional to $\Gamma_{(10)}$. The Weyl supersymmetry charges $\bar{Q}_{\alpha}^{+}$close on $Z_{(2)}^{+}$and $Z_{(6)}^{+}$. The $\tilde{F}$ commutations in terms of the $\mathrm{OSp}(1 \mid 32)$ and $\mathrm{Sp}(32)$ generators are given in appendix $\mathrm{B}$.

$\tilde{\boldsymbol{F}}$ transformation laws. Restricting the gauge parameter $\boldsymbol{\epsilon}$ to the $\tilde{F}$ subalgebra as described above, from (4.3) we deduce the $\tilde{F}$ transformation laws on the fields $B^{(n)}$ $(n=1,2,5,6,9,10)$ and $\psi$ :

$$
\begin{aligned}
& \delta B^{(\bar{n})}=d \varepsilon^{(\bar{n})}+\frac{\bar{n} !}{\bar{r} ! \bar{s} !} c_{(\bar{r})(\bar{s})}^{(\bar{n})} \varepsilon^{(\bar{r})} B^{(\bar{s})}-\frac{1}{16} \bar{\psi} \Gamma^{(\bar{n})} \epsilon \equiv D^{\tilde{F}} \varepsilon^{(\bar{n})}-\frac{1}{16} \bar{\psi}_{+} \Gamma^{(\bar{n})} \epsilon \\
& \delta B^{(\dot{n})}=\frac{\dot{n} !}{\bar{r} ! \dot{s} !} c_{(\bar{r})(\dot{s})}^{(\dot{s})} \varepsilon^{(\bar{r})} B^{(\dot{s})}+\frac{1}{16} \bar{\psi}_{-} \Gamma^{(\dot{n})} \epsilon \\
& \delta \psi_{+}=d \epsilon-\frac{1}{2 \cdot \bar{n} !} B^{(\bar{n})} \Gamma_{(\bar{n})} \epsilon+\frac{1}{2 \cdot \bar{n} !} \varepsilon^{(\bar{n})} \Gamma_{(\bar{n})} \psi_{+} \equiv D^{\tilde{F}} \epsilon+\frac{1}{2 \cdot \bar{n} !} \varepsilon^{(\bar{n})} \Gamma_{(\bar{n})} \psi_{+} \\
& \delta \psi_{-}=-\frac{1}{2 \cdot \dot{n} !} B^{(\dot{n})} \Gamma_{(\dot{n})} \epsilon+\frac{1}{2 \cdot \bar{n} !} \varepsilon^{(\bar{n})} \Gamma_{(\bar{n})} \psi_{-}
\end{aligned}
$$


where $\epsilon$ is the Weyl projected supersymmetry parameter, indices with a bar run on even values, and indices with a dot on odd values, i.e. $\bar{n}=2,6,10$ and $\dot{n}=1,5,9$. Moreover $\psi_{+}$and $\psi_{-}$are respectively Weyl and anti-Weyl projections of the Majorana gravitino, i.e. $\psi_{ \pm}=P_{ \pm} \psi$.

Thus we see that the $\tilde{F}$ gauge fields $B^{\bar{n}}, \psi_{+}$transform with the $\tilde{F}$ covariant derivative of the gauge parameters, whereas the "matter fields" $B^{\dot{n}}, \psi_{-}$transform homogeneously. These last include the vielbein $V=B^{(1)}$. Note also that gauge and matter fields do not mix, separating into a gauge and a matter multiplet under $\tilde{F}$ transformations.

Einstein-Hilbert term. The Lagrangian 12 -form $S \operatorname{Tr}\left(\mathbf{R}^{6} \boldsymbol{\Gamma}\right)$ contains the usual Einstein-Hilbert term

$$
\mathcal{R}^{a_{1} a_{2}} \wedge V^{a_{3}} \wedge \cdots \wedge V^{a_{12}} \varepsilon_{a_{1}-a_{12}}
$$

where $\mathcal{R}$ is the Lorentz curvature

$$
\mathcal{R}^{a_{1} a_{2}}=d B^{a_{1} a_{2}}-B^{a_{1}}{ }_{c} \wedge B^{c a_{2}}
$$

Indeed $\mathbf{R}^{6} \boldsymbol{\Gamma}$ includes a term (in the upper left corner of the supermatrix):

$$
\mathcal{R}^{a_{1} a_{2}} \wedge V^{a_{3}} \wedge V^{a_{4}} \wedge \cdots \wedge V^{a_{11}} \wedge V^{a_{12}} \Gamma_{a_{1} a_{2}} \Gamma_{a_{3} a_{4}} \cdots \Gamma_{a_{11} a_{12}} \Gamma_{13}
$$

Tracing on spinor indices produces the EH term (4.12), since $\operatorname{Tr}\left[\Gamma_{a_{1} a_{2}} \Gamma_{a_{3} a_{4}} \cdots \Gamma_{a_{11} a_{12}} \Gamma_{13}\right]$ is proportional to $\varepsilon_{a_{1}-a_{12}}$.

Field equations. They are obtained by varying the $\operatorname{OSp}(1 \mid 64)$ superconnection $\boldsymbol{\Omega}$ in (4.1), yielding:

$$
\delta S=\int S \operatorname{Tr}\left[\left\{(D \delta \boldsymbol{\Omega}) \mathbf{R}^{5}\right\} \boldsymbol{\Gamma}\right]=0
$$

where we have set for short

$$
\left\{(D \delta \boldsymbol{\Omega}) \mathbf{R}^{5}\right\} \equiv(D \delta \boldsymbol{\Omega}) \mathbf{R}^{5}+\mathbf{R}(D \delta \boldsymbol{\Omega}) \mathbf{R}^{4}+\cdots+\mathbf{R}^{5}(D \delta \boldsymbol{\Omega})
$$

and $D$ is the $\operatorname{OSp}(1 \mid 64)$ covariant derivative. Integrating by parts and using

$$
D \boldsymbol{\Gamma}=-\boldsymbol{\Omega} \boldsymbol{\Gamma}+\boldsymbol{\Gamma} \boldsymbol{\Omega}=\left(\begin{array}{cc}
{\left[\Gamma_{13}, \Omega\right]} & \left(\Gamma_{13}-1\right) \psi \\
\bar{\psi}\left(\Gamma_{13}-1\right) & 0
\end{array}\right)
$$

leads to

$$
\delta S=-2 \int S \operatorname{Sr}\left[\left\{\delta \boldsymbol{\Omega} \mathbf{R}^{5}\right\}\left(\begin{array}{cc}
\Omega^{(\text {odd })} \Gamma_{13} & \psi_{-} \\
\bar{\psi}_{-} & 0
\end{array}\right)\right]=0
$$

where $\Omega^{\text {(odd) }}$ contains only odd gamma matrices. Further analysis of the field equations (4.18), of their solutions and of possible "compactifications" relating to lower dimensional supergravities, notably $D=10,2 \mathrm{~b}$ supergravity, will be reported in a subsequent paper. 


\section{Conclusions}

The $D=12$ supergravity action we have presented here is made out of the fields contained in the $\operatorname{OSp}(1 \mid 64)$ connection, but is invariant only under a subalgebra $\tilde{F}$ of $\operatorname{OSp}(1 \mid 64)$. This closely resembles what happens for the Mac Dowell-Mansouri action in $D=4$, where the supergavity fields are organized in a $\operatorname{OSp}(1 \mid 4)$ connection, but the action itself is invariant only under the Lorentz subalgebra. In the $D=12$ case, however, supersymmetry is part of the invariance subalgebra of the action, since a Majorana-Weyl supercharge is included in $\tilde{F}$. This supercharge can give rise to at most $N=8$ supersymmetries once the action is reduced to four dimensions.

The constructive procedure adopted in this paper can be exported to the case of $D=9+1$ supergravity, for which a Majorana-Weyl fermion can be defined. The relevant "starting" superalgebra is $\operatorname{OSp}(1 \mid 32)$, generated by $Z^{(n)}$ with $n=(1,2,5,6,9,10)$ antisymmetric $D=10$ Lorentz indices, and a Majorana charge. The same argument of section 4 leads to an action $\int S \operatorname{Tr}\left(\mathbf{R}^{5} \boldsymbol{\Gamma}\right)$ where $\boldsymbol{\Gamma}$ involves $\Gamma_{11}$. This action is then invariant under the subalgebra of $\operatorname{OSp}(1 \mid 32)$ generated by the $Z^{(n)}$ with $n=(2,6,10)$ and the Weyl-projected Majorana charge. In this case the gauge multiplet is given by the one forms $B^{(2)}$ (spin connection), $B^{(6)}, B^{(10)}$ and $\psi_{+}=\frac{1}{2}\left(1+\Gamma_{11}\right) \psi$, and the matter multiplet by the remaining $B^{(1)}$ (vielbein), $B^{(5)}, B^{(9)}$ and $\psi_{-}=\frac{1}{2}\left(1-\Gamma_{11}\right) \psi$.

\section{A $\quad \mathrm{D}=12 \Gamma$ matrices, $\mathrm{Sp}(64)$ algebra and a Fierz identity}

\section{Clifford algebra.}

$$
\left\{\Gamma_{a}, \Gamma_{b}\right\}=2 \eta_{a b}, \quad \eta_{a b}=(+1,+1,-1, \cdots,-1), \quad \Gamma_{13}=\Gamma_{1} \cdots \Gamma_{12}, \quad \Gamma_{13}^{2}=1
$$

The antisymmetrizations of $n$ Lorentz indices in $\Gamma_{(n)}$ have weight 1 . For ex. $\Gamma_{a b} \equiv \frac{1}{2}\left[\Gamma_{a}, \Gamma_{b}\right]$. The $D=12$ charge conjugation matrix $C$ satisfies

$$
\Gamma_{a}^{T}=-C \Gamma_{a} C^{-1}, \quad C^{T}=-C
$$

so that $C \Gamma_{(n)}$ with $n=1,2,5,6,9,10$ are symmetric, and the remaining $C \Gamma_{(n)}$ with $n=$ $3,4,7,8,11,12$ are antisymmetric.

\section{Duality relations.}

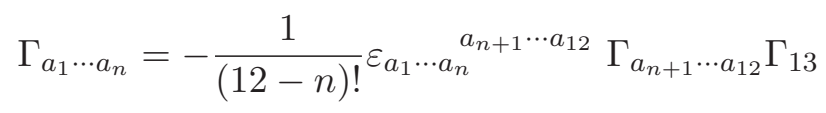

or, in a short-hand notation:

$$
\Gamma_{(n)}=-\frac{1}{(12-n) !} \varepsilon_{(n)}^{(12-n)} \Gamma_{(12-n)} \Gamma_{13}
$$

Defining the Weyl projectors

$$
P_{ \pm}=\frac{1}{2}\left(1 \pm \Gamma_{13}\right)
$$


one finds for example

$$
\begin{aligned}
\Gamma_{(10)} P_{ \pm} & =\mp \frac{1}{2} \varepsilon_{(10)}^{(2)} \Gamma_{(2)} P_{ \pm} \\
\Gamma_{(6)} P_{ \pm} & =\mp \frac{1}{6 !} \varepsilon_{(6)}{ }^{(6)} \Gamma_{(6)} P_{ \pm}
\end{aligned}
$$

The algebra of $\Gamma_{(n)}$.

$$
\left[\Gamma_{(n)}, \Gamma_{(m)}\right]=2 c_{(n)(m)}^{(r)} \Gamma_{(r)}
$$

The $\Gamma_{(n)}$ with $n=1,2,5,6,9,10$ generate the $\operatorname{Sp}(64)$ algebra. This algebra contains $\operatorname{Sp}(32) \oplus$ $\mathrm{Sp}(32)$, generated by the even $\Gamma^{\prime} \mathrm{s}: \Gamma_{(2)}, \Gamma_{(6)}$ and $\Gamma_{(10)}$. The two $\operatorname{Sp}(32)$ are separately generated by $\Gamma_{(2)} P_{+}, \Gamma_{(6)} P_{+}$, and by $\Gamma_{(2)} P_{-}, \Gamma_{(6)} P_{-}$. Note that $\Gamma_{(10)} P_{ \pm}$is proportional to $\Gamma_{(2)} P_{ \pm}$ because of (A.6) and that $\Gamma_{(6)} P_{+}$and $\Gamma_{(6)} P_{-}$are respectively selfdual and anti-selfdual.

The explicit form of the commutations (A.8) is:

$$
\left.\left[\Gamma^{a_{1}-a_{n}}, \Gamma_{b_{1}-b_{m}}\right]=2 \sum_{k=0}^{\min [m, n]} C_{n, m}^{n+m-2 k} \delta_{\left[b_{1}-b_{k}\right.}^{\left[a_{1}-a_{k}\right.} \Gamma^{\left.a_{k+1}-a_{n}\right]} b_{k+1}-b_{m}\right]
$$

where the structure constants $C_{n, m}^{r}$ are given in the following table:

\begin{tabular}{|c|c|c|c|c|c|c|c|c|c|c|c|}
\hline$n$ & $m$ & $r$ & $C_{n, m}^{r}$ & $n$ & $m$ & $r$ & $C_{n, m}^{r}$ & $n$ & $m$ & $r$ & $C_{n, m}^{r}$ \\
\hline 1 & 1 & 2 & 1 & 5 & 5 & 2 & $5 \cdot 5 !$ & 6 & 10 & 10 & $20 \cdot 6 !$ \\
\hline 1 & 2 & 1 & 2 & 5 & 6 & 9 & 30 & 6 & 10 & 6 & $-9 ! / 2$ \\
\hline 1 & 5 & 6 & 6 & 5 & 6 & 5 & $-10 \cdot 5 !$ & 9 & 9 & 10 & $21 \cdot 9 ! / 20$ \\
\hline 1 & 6 & 5 & 6 & 5 & 6 & 1 & $6 !$ & 9 & 9 & 6 & $-2 \cdot 7 \cdot 9 !$ \\
\hline 1 & 9 & 10 & 1 & 5 & 9 & 10 & $-6 !$ & 9 & 9 & 2 & $9 \cdot 9 !$ \\
\hline 1 & 10 & 9 & 10 & 5 & 9 & 6 & $3 \cdot 7 !$ & 9 & 10 & 9 & $21 \cdot 9 ! / 2$ \\
\hline 2 & 2 & 2 & -4 & 5 & 10 & 9 & $-10 \cdot 6 !$ & 9 & 10 & 5 & $-6 \cdot 10 !$ \\
\hline 2 & 5 & 5 & -10 & 5 & 10 & 5 & $6 \cdot 7 !$ & 9 & 10 & 1 & $10 !$ \\
\hline 2 & 6 & 6 & -12 & 6 & 6 & 10 & -36 & 10 & 10 & 10 & $-21 \cdot 9 !$ \\
\hline 2 & 9 & 9 & -18 & 6 & 6 & 6 & $20 \cdot 5 !$ & 10 & 10 & 6 & $20 \cdot 10 !$ \\
\hline 2 & 10 & 10 & -20 & 6 & 6 & 2 & $-6 \cdot 6 !$ & 10 & 10 & 2 & $-10 \cdot 10 !$ \\
\hline 5 & 5 & 10 & 1 & 6 & 9 & 9 & $8 ! / 4$ & & & & \\
\hline 5 & 5 & 6 & -200 & 6 & 9 & 5 & $-9 ! / 4$ & & & & \\
\hline
\end{tabular}

Fierz identity. The following Fierz identity holds for Majorana spinor one-forms $(\bar{\psi}=$ $\psi C)$ :

$$
\psi \bar{\psi}=\frac{1}{64} \sum_{n} \frac{1}{n !} \bar{\psi} \Gamma^{(n)} \psi \Gamma_{(n)} \quad n=1,2,5,6,9,10
$$

where for example $\bar{\psi} \Gamma^{(2)} \psi \Gamma_{(2)}$ is defined by $\bar{\psi} \Gamma^{a_{1} a_{2}} \psi \Gamma_{a_{1} a_{2}}$ etc. 


\section{B The $\tilde{\boldsymbol{F}}$ superalgebra}

The $\tilde{F}$ generators satisfy the following (anti)commutation relations:

$$
\begin{array}{rlrl}
{\left[Z_{(2)}^{ \pm}, Z_{(2)}^{ \pm}\right]} & =c_{(2)(2)}^{(2)} Z_{(2)}^{ \pm} & \\
{\left[Z_{(2)}^{ \pm}, Z_{(6)}^{ \pm}\right]} & =c_{(2)(2)}^{(6)} Z_{(6)}^{ \pm} & \\
{\left[Z_{(6)}^{ \pm}, Z_{(6)}^{ \pm}\right]} & =\left[c_{(6)(6)}^{(2)}-\frac{1}{2} c_{(6)(6)}^{(10)} \varepsilon_{(10)}^{(2)}\right] Z_{(2)}^{ \pm}+c_{(6)(6)}^{(6)} Z_{(6)}^{ \pm} & \\
{\left[Z_{(r)}^{ \pm}, Z_{(s)}^{\mp}\right]=0,} & r, s=2,6 \\
\left\{\bar{Q}_{\alpha}^{+}, \bar{Q}_{\beta}^{+}\right\} & =\frac{1}{16 \cdot n !}\left(C \Gamma^{(n)} P_{+}\right)_{\alpha \beta} Z_{(n)}^{+}, & n=2,6 \\
{\left[Z_{(n)}^{+}, \bar{Q}_{\alpha}^{+}\right]} & =\frac{1}{2}\left(\Gamma_{(n)} P_{+}\right)^{\beta}{ }_{\alpha} \bar{Q}_{\beta}^{+}, & n=2,6 \\
{\left[Z_{(n)}^{-}, \bar{Q}_{\alpha}^{+}\right]} & =0, & n=2,6
\end{array}
$$

The generators with a + superscript belong to $\operatorname{OSp}(1 \mid 32)$, those with a - superscript to $\operatorname{Sp}(32)$.

Open Access. This article is distributed under the terms of the Creative Commons Attribution License (CC-BY 4.0), which permits any use, distribution and reproduction in any medium, provided the original author(s) and source are credited.

\section{References}

[1] L. Castellani, P. Fré, F. Giani, K. Pilch and P. van Nieuwenhuizen, Beyond $d=11$ supergravity and Cartan integrable systems, Phys. Rev. D 26 (1982) 1481 [INSPIRE].

[2] D. Kutasov and E.J. Martinec, New principles for string/membrane unification, Nucl. Phys. B 477 (1996) 652 [hep-th/9602049] [INSPIRE].

[3] I. Bars, Supersymmetry, p-brane duality and hidden space-time dimensions, Phys. Rev. D 54 (1996) 5203 [hep-th/9604139] [InSPIRE].

[4] I. Bars, S theory, Phys. Rev. D 55 (1997) 2373 [hep-th/9607112] [InSPIRE].

[5] S. Hewson and M. Perry, The twelve-dimensional super $(2+2)$-brane, Nucl. Phys. B 492 (1997) 249 [hep-th/9612008] [INSPIRE].

[6] A.A. Tseytlin, Type IIB instanton as a wave in twelve-dimensions, Phys. Rev. Lett. 78 (1997) 1864 [hep-th/9612164] [INSPIRE].

[7] T. Hurth, P. van Nieuwenhuizen, A. Waldron and C. Preitschopf, On a possible new $R^{2}$ theory of supergravity, Phys. Rev. D 55 (1997) 7593 [hep-th/9702052] [InSPIRE].

[8] N. Khviengia, Z. Khviengia, H. Lü and C.N. Pope, Towards a field theory of F-theory, Class. Quant. Grav. 15 (1998) 759 [hep-th/9703012] [INSPIRE].

[9] I. Bars and C. Kounnas, Theories with two times, Phys. Lett. B 402 (1997) 25 [hep-th/9703060] [INSPIRE].

[10] H. Nishino, $N=2$ chiral supergravity in $(10+2)$-dimensions as consistent background for super(2 + 2)-brane, Phys. Lett. B 437 (1998) 303 [hep-th/9706148] [INSPIRE]. 
[11] I. Rudychev, E. Sezgin and P. Sundell, Supersymmetry in dimensions beyond eleven, Nucl. Phys. Proc. Suppl. 68 (1998) 285 [hep-th/9711127] [INSPIRE].

[12] R. Manvelyan and R. Mkrtchian, Towards $\mathrm{SO}(2,10)$ invariant M-theory: multiLagrangian fields, Mod. Phys. Lett. A 15 (2000) 747 [hep-th/9907011] [INSPIRE].

[13] S. Hewson, On supergravity in $(10,2)$, hep-th/9908209 [INSPIRE].

[14] C. Vafa, Evidence for F-theory, Nucl. Phys. B 469 (1996) 403 [hep-th/9602022] [inSPIRE].

[15] S.J. Gates, M.T. Grisaru, M. Roček and W. Siegel, Superspace or one thousand and one lessons in supersymmetry, Front. Phys. 58 (1983) 1 [hep-th/0108200] [INSPIRE].

[16] J. Wess and J. Bagger, Supersymmetry and supergravity, Princeton Univ. Pr., Princeton U.S.A., (1992) [INSPIRE].

[17] D.Z. Freedman and A. Van Proeyen, Supergravity, Cambridge University Press, Cambridge U.K., (2012) [INSPIRE].

[18] L. Castellani, R. D'Auria and P. Fré, Supergravity and superstrings: a geometric perspective. Volume 1: mathematical foundations, World Scientific, Singapore, (1991) [INSPIRE].

[19] L. Castellani, R. D'Auria and P. Fré, Supergravity and superstrings: a geometric perspective. Volume 2: supergravity, World Scientific, Singapore, (1991) [INSPIRE].

[20] L. Castellani, R. D'Auria and P. Fré, Supergravity and superstrings: a geometric perspective. Volume 3: superstrings, World Scientific, Singapore, (1991) [INSPIRE].

[21] L. Castellani, P. Fré and P. van Nieuwenhuizen, A review of the group manifold approach and its application to conformal supergravity, Annals Phys. 136 (1981) 398 [INSPIRE].

[22] L. Castellani, Group geometric methods in supergravity and superstring theories, Int. J. Mod. Phys. A 7 (1992) 1583 [inSPIRE].

[23] J. Zanelli, Lecture notes on Chern-Simons (super-)gravities. Second edition (February 2008), hep-th/0502193 [INSPIRE].

[24] P. Van Nieuwenhuizen, Supergravity, Phys. Rept. 68 (1981) 189 [InSPIRE].

[25] L. Castellani, R. Catenacci and P.A. Grassi, The integral form of supergravity, JHEP 10 (2016) 049 [arXiv: 1607.05193] [INSPIRE].

[26] S.W. MacDowell and F. Mansouri, Unified geometric theory of gravity and supergravity, Phys. Rev. Lett. 38 (1977) 739 [Erratum ibid. 38 (1977) 1376] [INSPIRE].

[27] A.H. Chamseddine and P.C. West, Supergravity as a gauge theory of supersymmetry, Nucl. Phys. B 129 (1977) 39 [InSPIRE].

[28] P. van Nieuwenhuizen, Supergravity as a Yang-Mills theory, in 50 years of Yang-Mills theory, G. 't Hooft ed., World Scientific, Singapore, (2005), pg. 433 [hep-th/0408137] [INSPIRE].

[29] R. Troncoso and J. Zanelli, Gauge supergravities for all odd dimensions, Int. J. Theor. Phys. 38 (1999) 1181 [hep-th/9807029] [INSPIRE].

[30] L. Castellani, $\mathrm{OSp}(1 \mid 4)$ supergravity and its noncommutative extension, Phys. Rev. D 88 (2013) 025022 [arXiv:1301.1642] [INSPIRE]. 